\title{
ON UNIVERSAL COMPOSITIONS OF MAPS
}

\author{
ZVONKO ČERIN*
}

\begin{abstract}
In this paper we shall introduce notions of $\mathcal{F}$-universality and $\mathcal{F}$-e-universality for maps between compact Hausdorf spaces and explore the behaviour of these properties under the operation of composition of maps. We consider both the quest for conditions on maps $f$ and $g$ which would imply that their composition $g \circ f$ is either $\mathcal{F}$-universal or $\mathcal{F}$-e-universal and the quest for consequences on $f$ and $g$ when the composition $g \circ f$ is either $\mathcal{F}$-universal or $\mathcal{F}$-e-universal. In our approach $\mathcal{F}$ is an arbitrary class of maps. For a special choice of $\mathcal{F}$, the notion of $\mathcal{F}$-universality reduces to Holsztyniski's notion of universality while $\mathcal{F}$-e-universality reduces to Sanjurjo's notion of proximate universality.
\end{abstract}

Throughout the paper, unless stated otherwise, by a space we mean a compact Hausdorff space and by a map we mean a continuous function between spaces. We shall always consider maps $f: X \rightarrow Y$ and $g: Y \rightarrow Z$ and their composition $g \circ f: X \rightarrow Z$. The letters $M, N$, and $P$ will be reserved for spaces containing $X, Y$, and $Z$ as closed subsets, respectively.

We shall use $\mathcal{F}$ and $\mathcal{G}$ to denote arbitrary classes of maps. The fact that a map $f$ belongs to $\mathcal{F}$ will be expressed by saying that $f$ is an $\mathcal{F}_{\text {-map. Let }} \mathcal{F}_{A}^{Z}$ denote the class of all $\mathcal{F}$-maps $a: A \rightarrow B$ with $B$ contained in $Z$. Let $\mathcal{A}$ be the class of all maps.

For maps $a: X \rightarrow Y$ and $b: X \rightarrow Y$ between spaces $X$ and $Y$ and an open cover $\sigma$ of $Y$ we let $a \approx b, a \stackrel{\sigma}{\approx} b$, and $a \stackrel{\sigma}{=} b$ mean that $a(x)=b(x)$ for some $x \in X, a(x) \stackrel{\sigma}{=} b(x)$ (i. e., that some member of $\sigma$ contains both $a(x)$ and $b(x))$ for some $x \in X$, and $a(x) \stackrel{\sigma}{=} b(x)$ for every $x \in X$, respectively.

A map $f: X \rightarrow Y$ is $\mathcal{F}$-universal provided $f \approx a$ for every $\mathcal{F}_{X}^{Y}$-map $a$. Observe that a map is $\mathcal{A}$-universal iff it is universal in the sense of Holsztyński [5].

In order to define $\mathcal{F}$-e-universal maps we shall use Borsuk's method from [2]. In this approach we do not require exact coincidence and we allow that maps slip outside of compacta into neighborhoods in an ambient space.

*Dedicated to Chelo 
Let $\bar{X}$ denote the collection of all open covers of a space $X$. Let $n(A, X)$ and $k n(A, X)$ stand for the collection of all open and of all compact neighborhoods in $X$ of a subset $A$ in $X$, respectively. Let $\bar{X}_{A}$ denote all collections of open subsets of $X$ which cover $A$ and let $i_{A, X}$ be the inclusion of $A$ into $X$.

A map $f: X \rightarrow Y$ is $\mathcal{F}$-e-universal in $N$ provided for every $\sigma \in \tilde{N}_{Y}$ there is a $U \in n(Y, N)$ such that $f \stackrel{\sigma}{\approx} a$ for every $a \in \mathcal{F}_{X}^{U}$. Observe that a map of metric compacta is $\mathcal{A}$-e-universal in the Hilbert cube $Q$ iff it is proximately universal in the sense of Sanjurjo [10, Theorem 5].

It was already noticed by Holsztynski in [7] that the composition of universal maps need not be universal. Some partial results in the identification of sufficient conditions on maps $f: X \rightarrow Y$ and $g: Y \rightarrow Z$ which imply that the composition $g \circ f: X \rightarrow Z$ is (proximately) universal are included in [6], [7], [1], and [10].

In the present paper we establish theorems which give answers to the above problem for $\mathcal{F}$-universality and $\mathcal{F}$-e-universality in $N$ and also to a question to find conditions which imply that either $f$ or $g$ is $\mathcal{F}$-universal and $\mathcal{F}$-e-universal in $N$ and in $P$ when the composition $g \circ f$ is $\mathcal{F}$-universal and $\mathcal{F}$-e-universal in $P$, respectively.

Our first result shows that in the definition of universalities with respect to a class $\mathcal{F}$ we can always pass on to a larger class.

A map $f$ is an $[\mathcal{F}]$-map provided for every $\sigma \in \tilde{Y}$ there is an $a \in \mathcal{F}_{X}^{Y}$ with $f \stackrel{\sigma}{=} a$. It is an $[\mathcal{F}, N)$-map provided for every $\sigma \in \tilde{N}_{Y}$ and every $V \in n(Y, N)$ there is an $a \in \mathcal{F}_{X}^{V}$ with $f \stackrel{\sigma}{=} a$. Similarly, $f$ is an $(\mathcal{F}, M, N)$-map provided for every $\sigma \in \tilde{N}_{Y}$ and every $V \in n(Y, N)$ there is a $U \in k n(X, M)$ and an $a \in \mathcal{F}_{U}^{V}$ with $\left.f \stackrel{o}{=} a\right|_{X}$.

\section{Theorem 1.}

(a) A map $f$ is $\mathcal{F}$-universal iff it is $[\mathcal{F}]$-universal.

(b) A map $f$ is $\mathcal{F}$-e-universal in $N$ iff it is $[\mathcal{F}, N)$-e-universal in $N$.

Proof: (b). Let $\xi \in \tilde{N}_{Y}$. Let $\xi^{*}$ and $\xi^{* *}$ denote the set of all members $\sigma$ of $\tilde{N}_{Y}$ such that the star of $\sigma$ and the double-star of $\sigma$ refines $\xi$, respectively. Let $\sigma \in \xi^{*}$. By assumption, there is a $V \in n(Y, N)$ such that $f \stackrel{\approx}{\approx} h$ for every $h \in \mathcal{F}_{X}^{V}$.

Consider a $k \in[\mathcal{F}, N]_{X}^{V}$. Choose an $h \in \mathcal{F}_{X}^{V}$ with $h \stackrel{\sigma}{=} k$. Since $f \stackrel{\sigma}{\approx} h$, we get $f \stackrel{\xi}{\approx} k$.

The following theorem resembles Theorem (3.3) in [7] and Theorem 9 in [10].

For a map $f: X \rightarrow Y$ and a space $Z$, let $\sigma_{Z}(f): X \times Z \rightarrow Z \times Y$ be a map defined by $\sigma_{Z}(f)(x, z)=(z, f(x))$ for every $(x, z) \in X \times Z$. Let $\sigma_{Z}(\mathcal{F})=\left\{\sigma_{Z}(f): f \in \mathcal{F}\right\}$. 


\section{Theorem 2.}

(a) The composition $g \circ f$ is $\mathcal{F}$-universal iff the product $f \times g$ is $\sigma_{Y}(\mathcal{F})$ universal.

(b) The composition $g \circ f$ is $\mathcal{F}$-e-universal in $P$ iff the product $f \times g$ is $\sigma_{Y}(\mathcal{F})$-e-universal in $Y \times P$.

Proof: (b). Let $g \circ f$ be $\mathcal{F}$-e-universal in $P$. Let $\xi \in \widetilde{Y \times P Y \times Z}$. Choose an $\eta \in \tilde{P}_{Z}$ such that $b \stackrel{\underline{\eta}}{=} c$ in $P$ implies $(a, b) \stackrel{\xi}{=}(a, c)$ in $Y \times P$ for every $a \in Y$. By assumption, there is a $V \in n(Z, P)$ with $h \stackrel{\eta}{\approx} g \circ f$ for every $h \in \mathcal{F}_{X}^{V}$. Let $U=Y \times V \in n(Y \times Z, Y \times P)$.

Consider a $k \in \sigma_{Y}(\mathcal{F})_{X \times Y}^{U}$. Pick an $h \in \mathcal{F}_{X}^{V}$ such that $k=\sigma_{Y}(h)$. The way in which $V$ was selected implies the existencc of an $x \in X$ with $h(x) \stackrel{\underline{n}}{=} g \circ f(x)$. Then

$$
k(x, f(x))=\sigma_{Y}(h)(x, f(x))=(f(x), h(x))
$$

and $(f(x), h(x)) \stackrel{\underline{\xi}}{=}(f(x), g \circ f(x))=(f \times g)(x, f(x))$. Hence, $k \stackrel{\xi}{\approx} f \times g$.

Conversely, suppose that $f \times g$ is $\sigma_{Y}(\mathcal{F})$-e-universal in $Y \times P$. Let $\eta \in \tilde{P}_{Z}$. Let $\mu \in \eta^{*}$. Let $\theta=g^{-1}(\mu)$. Let $\xi=\{E \times M: E \in \theta, M \in \mu\} \in \widehat{Y \times P} \times P_{\times Z}$. By assumption, there is a $U \in n(Y \times Z, Y \times P)$ such that $k \stackrel{\xi}{\approx} f \times g$ for every $k \in \sigma_{Y}(\mathcal{F})_{X \times Y}^{U}$. Choose a $V \in n(Z, P)$ with $Y \times V \subset U$.

Consider an $h \in \mathcal{F}_{X}^{V}$. Since $\sigma_{Y}(h)$ is in $\sigma_{Y}(\mathcal{F})_{X \times Y}^{U}$, there is an $(x, y) \in X \times Y$ with $(y, h(x)) \stackrel{\underline{\xi}}{=}(f(x), g(y))$. In other words, $y \stackrel{\theta}{=} f(x)$ and $h(x) \stackrel{\mu}{=} g(y)$. Hence, $h \stackrel{\eta}{\approx} g \circ f$

The first half of the above theorem clearly includes Theorem (3.3) in [7]. In order to see that it also generalizes Theorcm 9 in $[\mathbf{1 0}]$, we need the following theorem.

Recall [9] that a space $X$ is an approximate polyhedron provided for evcry $\sigma \in \tilde{X}$ there is a polyhedron $K$ and maps $u: X \rightarrow K$ and $d: K \rightarrow X$ with $d \circ u \stackrel{\sigma}{=} i d_{X}$, where $i d_{X}$ is the identity map on $X$. Onc can easily see that a space is an approximate polyhedron iff it is an approximate absolute ncighborhood retract (in the sense of Clapp) for the class of all compact Hausdorff spaces [4].

A class $\mathcal{F}$ of maps is solid provided $f \circ g \in \mathcal{F}$ for every $f \in \mathcal{F}$ and every map $g$ such that $f \circ g$ can be defined. Similarly, $\mathcal{F}$ is a legal class provided $f \circ g \in \mathcal{F}$ for every $g \in \mathcal{F}$ and every map $f$ such that $f \circ g$ can be defined. Clearly, the class $\mathcal{A}$ is both solid and legal.

Theorem 3. Let $\mathcal{F}$ be a legal class of maps. If $f$ is $\mathcal{F}$-e-universal in some

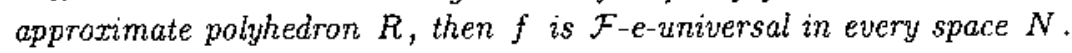

Proof: Let $\sigma \in \hat{N}_{Y}$. Let $T$ be a space obtained by glueing $R$ and $N$ along $Y$. Since $N$ is a closed subset of $T$, there is an $\eta \in T_{Y}$ such that $\left.\eta\right|_{N}$ refines 
$\sigma$. Let $\xi \in \eta^{*}$. By assumption, there is a $V \in n(Y, R)$ with $f \stackrel{\xi}{\approx} h$ for every $h \in \mathcal{F}_{X}^{V}$. Select refinements $\pi$ of $\left.\xi\right|_{R}$ and $\rho$ of $\xi$ such that the star $\operatorname{st}(Y, \pi)$ of $Y$ with respect to the collection $\pi$ is a subset of $V$ and that $\left.\rho\right|_{R}$ refines $\pi$. Let $\tau \in \rho^{*}$. Since $R$ is an approximate polyhedron, there is an $S \in n(R, T)$ and a map $r: S \rightarrow R$ such that $y \stackrel{\tau}{=} r(y)$ for every $y \in Y$. Hence, there is a $U \in n(Y, N)$ such that $U \subset S \bigcap s t(Y, \tau)$ and $u \stackrel{\tau}{=} r(u)$ for every $u \in U$.

Consider an $h \in \mathcal{F}_{X}^{U}$. Let $k=r \circ h$. Since $\mathcal{F}$ is legal, $k \in \mathcal{F}$. But, $k(X) \subset \operatorname{st}\left(Y,\left.\rho\right|_{R}\right) \subset \operatorname{st}(Y, \pi) \subset V$. Hence, $k \stackrel{\xi}{\approx} f$. It follows that $f \stackrel{\eta}{\approx} h$ because $k \stackrel{\tau}{\approx} h$. Since $f(X) \bigcup h(X) \subset N$, we get $f \stackrel{\sigma}{\approx} h$.

The next three results are related to Proposition (3.9) in [1] and the first half of Theorem 8 in [10].

A map $g$ is an $X[\mathcal{F}, \mathcal{G}]$-e-retraction provided for every $\sigma \in \tilde{Z}$ and every $a \in \mathcal{F}_{X}^{Z}$ there is a $b \in \mathcal{G}_{X}^{Y}$ with $a \stackrel{\sigma}{=} g \circ b$.

A map $g$ is an $X(\mathcal{F}, \mathcal{G})$-e-retraction in $P$ provided for every $\sigma \in \tilde{P}_{Z}$ there is a $W \in n(Z, P)$ such that for every $a \in \mathcal{F}_{X}^{W}$ there is a $b \in \mathcal{G}_{X}^{Y}$ with $a \stackrel{\sigma}{=} g \circ b$.

A map $g$ is an $X(\mathcal{F}, \mathcal{G})$-e-retraction in $(N, P)$ provided for every $\sigma \in \bar{P}_{Z}$ there is a $U \in n(Y, N)$ and a map $G: U \rightarrow P$ such that $\left.g \stackrel{\sigma}{=} G\right|_{Y}$ and for every $V \in n(Y, U)$ there is a $W \in n(Z, P)$ so that for every $a \in \mathcal{F}_{X}^{W}$ there is a $b \in \mathcal{G}_{X}^{V}$ with $a \stackrel{o}{=} G \circ b$.

Observe that $\mathrm{g}$ is a $Z[\mathcal{A}, \mathcal{A}]$-e-retraction iff it is ARI (approximately right invertible) [8]. Also, $g$ is a $Z(\mathcal{A}, \mathcal{A})$-e-retraction in $(Q, Q)$ iff it is a weakly refinable map (see [10, Theorem 6$]$ ).

\section{Theorem 4.}

(a) If $f$ is $\mathcal{G}$-universal and $g$ is an $X[\mathcal{F}, \mathcal{G}]$-e-retraction, then $g \circ f$ is $\mathcal{F}$ universal.

(b) If $f$ is $\mathcal{G}$-universal and $g$ is an $X(\mathcal{F}, \mathcal{G})$-e-retraction in $P$, then $g \circ f$ is $\mathcal{F}$-e-universal in $P$.

(c) If $f$ is $\mathcal{G}$-e-universal in $N$ and $g$ is an $X(\mathcal{F}, \mathcal{G})$-e-retraction in $(N, P)$, then $g \circ f$ is $\mathcal{F}$-e-universal in $P$.

Proof: (c). Let $\sigma \in \hat{P}_{Z}$. Let $\eta \in \sigma^{*}$. Since $g$ is an $X(\mathcal{F}, \mathcal{G})$-e-retraction in $(N, P)$, there is a $U \in n(Y, N)$ and a map $G: U \rightarrow P$ such that $\left.g \stackrel{\eta}{=} G\right|_{y}$ and for every $V \in n(Y, U)$ there is a $W \in n(Z, P)$ so that for every $a \in \mathcal{F}_{X}^{W}$ there is a $b \in \mathcal{G}_{X}^{V}$ with $a \stackrel{\eta}{=} G \circ b$. Let $\theta=G^{-1}(\eta) \in \tilde{N}_{Y}$.

Since $f$ is $\mathcal{G}$-e-universal in $N$, there is an $S \in n(Y, N)$ with the property that $h \stackrel{\ominus}{\approx} f$ for every $h \in \mathcal{G}_{X}^{S}$. Let $V=S \bigcap U \in n(Y, U)$. By assumption, there is a $W \in n(Z, P)$ such that for every $a \in \mathcal{F}_{X}^{W}$ there is a $b \in \mathcal{G}_{X}^{V}$ with $a \stackrel{\eta}{=} G \circ b$.

Consider a $k \in \mathcal{F}_{X}^{W}$. Choose an $h \in \mathcal{G}_{X}^{V}$ with $k \stackrel{n}{=} G \circ h$. The way in which $V$ was selected implies $h \stackrel{\theta}{=} f$. Hence, $G \circ h \stackrel{\eta}{\approx} G \circ f \stackrel{\eta}{=} g \circ f$. Finally, we get 
$k \stackrel{a}{\approx} g \circ f$

A map $g$ is $A R I[\mathcal{F}]$ provided for every $\sigma \in \tilde{Z}$ there is an $s \in \mathcal{F}_{Z}^{Y}$ with $g \circ s \stackrel{\underline{\sigma}}{=} i d_{Z}$.

A map $g$ is $A R I(\mathcal{F})$ in $(N, P)$ provided for every $\sigma \in \tilde{P}_{Z}$ there is a $U \in$ $n(Y, N)$ and a map $G: U \rightarrow P$ such that $\left.g \stackrel{\sigma}{=} G\right|_{Y}$ and for every $V \in n(Y, U)$ there is an $s \in \mathcal{F}_{Z}^{V}$ with $G \circ s \stackrel{\sigma}{=} i_{Z, P}$.

A map $g$ is $A R I(\mathcal{F})$ in $(N, P)$ provided for every $\sigma \in \tilde{P}_{Z}$ there is a $U \in$ $n(Y, N)$, a map $G: U \rightarrow P$, and a $W \in k n(Z, P)$ such that $\left.g \stackrel{\sigma}{=} G\right|_{Y}$ and for every $V \in n(Y, U)$ there is an $s \in \mathcal{F}_{W}^{V}$ with $G \circ s \stackrel{\text { a }}{=} i_{W, P}$.

Clearly, $A R I[\mathcal{A})$ and $A R I(\mathcal{A})$ in $(Q, Q)$ maps agree with weakly refinable maps, while $A R I[\mathcal{A}]$ maps agree with ARI maps.

Theorem 5. Let $\mathcal{F}$ be a solid class of maps.

(a) If $f$ is $\mathcal{F}$-universal and $g$ is $A R I[\mathcal{F}]$, then $g \circ f$ is $\mathcal{A}$-universal.

(b) If $f$ is $\mathcal{F}$-e-universal in $N$ and $g$ is $A R I[\mathcal{F})$ in $(N, P)$, then $g \circ f$ is A-universal.

(c) If $f$ is $\mathcal{F}$-e-universal in $N$ and $g$ is $A R I(\mathcal{F})$ in $(N, P)$, then $g \circ f$ is $\mathcal{A}$-e-universal in $P$.

Proof: (c). Let $\sigma \in \tilde{P}_{Z}$. Let $\xi \in \sigma^{*}$. Since $g$ is $A R I(\mathcal{F})$ in $(N, P)$, there is a $V \in n(Y, N)$ and a map $G: V \rightarrow P$ such that $\left.g \stackrel{\xi}{=} G\right|_{Y}$ and for every $W \in n(Y, V)$ there is a $U \in k n(Z, P)$ and an $s \in \mathcal{F}_{U}^{W}$ with $G \circ s \stackrel{\xi}{=} i_{U, P}$.

Let $\theta=G^{-1}(\xi)$. Sclect a $W \in n(Y, V)$ such that $f \stackrel{\theta}{\approx} h$ for every $h \in \mathcal{F}_{X}^{W}$. By assumption, there is a $U \in k n(Z, P)$ and an $s \in \mathcal{F}_{U}^{W}$ with $G \circ s \underline{\underline{\xi}} i_{U, P}$.

Let $k: X \rightarrow$ int $U$ be a map. Observe that $s \circ k \in \mathcal{F}_{X}^{W}$ because $\mathcal{F}$ is a solid class of maps. Hence, $f \stackrel{\theta}{\approx} s \circ k, g \circ f \stackrel{\xi}{=} G \circ f \stackrel{\xi}{\approx} G \circ s \circ k \stackrel{\xi}{=} k$, and $k \stackrel{g}{\approx} g \circ f$.

In the statement of the next theorem we shall need the following notions. They could be regarded as dual to the notions of nearly extendable maps [2] (or, equivalently, e-movable maps [3]).

A map $f$ is $[\mathcal{F}, \mathcal{G}]$-e-liftable in $Z$ provided for every $\sigma \in \tilde{Z}$ and every $a \in \mathcal{F}_{Y}^{Z}$ there is a $b \in \mathcal{G}_{X}^{Z}$ with $b \stackrel{\sigma}{=} a \circ f$.

A map $f$ is $\left(\mathcal{F}, \mathcal{G}\right.$-e-liftable in $(P, Z)$ provided for every $\sigma \in \tilde{P}_{Z}$ there is a $W \in n(Z, P)$ such that for every $a \in \mathcal{F}_{Y}^{W}$ there is $a b \in \mathcal{G}_{X}^{Z}$ with $b \stackrel{\sigma}{=} a \circ f$.

A map $f$ is $[\mathcal{F}, \mathcal{G})$-e-liftable in $(P, Z)$ provided for every $\sigma \in \tilde{P}_{Z}$, every $U \in n(Z, P)$, and every $a \in \mathcal{F}_{Y}^{Z}$ there is a $b \in \mathcal{G}_{X}^{U}$ with $b \stackrel{\sigma}{=} a \circ f$.

A map $f$ is $(\mathcal{F}, \mathcal{G})$-e-liftable in $(P, Z)$ provided for every $\sigma \in \bar{P}_{Z}$ and every $V \in n(Z, P)$ there is a $W \in n(Z, P)$ such that for every $a \in \mathcal{F}_{Y}^{W}$ there is a $b \in \mathcal{G}_{X}^{V}$ with $b \stackrel{\sigma}{=} a \circ f$. 
Let $\lambda_{Z}[\mathcal{F}, \mathcal{G}]$ denote the class of all $[\mathcal{F}, \mathcal{G}]$-e-liftable in $Z$ maps. The notations $\lambda_{(P, Z)}(\mathcal{F}, \mathcal{G}], \lambda_{(P, Z)}[\mathcal{F}, \mathcal{G})$, and $\lambda_{(P, Z)}(\mathcal{F}, \mathcal{G})$ have analogous meanings.

A map $g$ is extendable in $(N, P)$ provided for every $\sigma \in \tilde{P}_{Z}$ there is a $U \in n(Y, N)$ and a map $G: U \rightarrow P$ such that $\left.g \stackrel{\sigma}{=} G\right|_{Y}$.

Theorem 6. Let $\mathcal{F}$ be a solid class of maps.

(a) If $f$ is $\mathcal{G}$-universal and $g$ is $A R I[\mathcal{F}]$, then $g \circ f$ is $\lambda_{Y}[\mathcal{F}, \mathcal{G}]$-universal.

(b) If $f$ is $\mathcal{G}$-e-universal in $N$ and $g$ is both $A R I[\mathcal{F}]$ and extendable in $(N, P)$, then $g \circ f$ is $\lambda_{(N, Y)}[\mathcal{F}, \mathcal{G})$-universal.

(c) If $f$ is $\mathcal{G}$-universal and $g$ is $A R I[\mathcal{F})$ in $(N, P)$, then gof is $\lambda_{(N, Y)}(\mathcal{F}, \mathcal{G}\}$ universal.

(d) If $f$ is $\mathcal{G}$-e-universal in $N$ and $g$ is $A R I[\mathcal{F})$ in $(N, P)$, then $g \circ f$ is $\lambda_{(N, Y)}(\mathcal{F}, \mathcal{G})$-universal.

(e) If $f$ is $\mathcal{G}$-e-universal in $N$ and $g$ is $A R I(\mathcal{F})$ in $(N, P)$, then $g \circ f$ is $\lambda_{(N, Y)}(\mathcal{F}, \mathcal{G})$-e-universal in $P$.

Proof: $(d)$. Let a $\sigma \in \tilde{Z}$ and a $\lambda_{(N, Y)}(\mathcal{F}, \mathcal{G})_{\text {-map }} k: X \rightarrow A$ with $A$ contained in $Z$ be given. Pick an $\eta \in \tilde{P}$ such that the restriction $\left.\eta\right|_{Z}$ of $\eta$ to $Z$ refines $\sigma$. Let $\xi \in \eta^{* *}$. Since $g$ is $A R I[\mathcal{F})$ in $(N, P)$, there is a $U \in n(Y, N)$ and a map $G: U \rightarrow P$ such that $\left.g \stackrel{\xi}{=} G\right|_{Y}$ and for every $V \in n(Y, U)$ there is an $s \in \mathcal{F}_{Z}^{V}$ with $G \circ s \stackrel{\xi}{=} i_{Z, P}$. Let $\theta=G^{-1}(\xi) \in \tilde{N}_{Y}$.

By assumption, there is a $V \in n(Y, U)$ such that $h \stackrel{\theta}{\approx} f$ for every $h \in \mathcal{G}_{X}^{V}$. Since $k$ is $(\mathcal{F}, \mathcal{G})$-e-liftable in $(N, P)$, there is a $W \in n(Y, U)$ with the property that that for every $a \in \mathcal{F}_{A}^{W}$ there is a $b \in \mathcal{G}_{X}^{V}$ with $b \stackrel{\theta}{=} a \circ k$. By assumption on $g$, there is an $s \in \mathcal{F}_{Z}^{W}$ with $G \circ s \stackrel{\underline{\xi}}{=} i_{Z, P}$. Choose a $t \in \mathcal{G}_{X}^{V}$ with $t \stackrel{\theta}{=}\left(\left.s\right|_{A}\right) \circ k$.

The way in which $V$ was selected implies $f \stackrel{\theta}{\approx} t$. Combining the above relations, we get $g \circ f \stackrel{\underline{\xi}}{=} G \circ f \stackrel{\xi}{\approx} G \circ t \stackrel{\underline{\xi}}{=} G \circ\left(\left.s\right|_{A}\right) \circ k \stackrel{\underline{\xi}}{=} i_{A, P} \circ k=k$. Hence, $k \stackrel{\sigma}{\approx} g \circ f$.

(e). Let $\sigma \in \tilde{P}_{Z}$. Let $\eta \in \sigma^{* *}$. Since $g$ is $A R I(\mathcal{F})$ in $(N, P)$, there is a $U \in n(Y, N)$, a map $G: U \rightarrow P$, and a $W \in k n(Z, P)$ such that $\left.g \stackrel{\eta}{=} G\right|_{Y}$ and for every $V \in n(Y, U)$ there is an $s \in \mathcal{F}_{W}^{V}$ with $G \circ s \stackrel{\underline{\eta}}{=} i_{W, P}$. Let $\theta=G^{-1}(\eta) \in$ $\tilde{N}_{Y}$.

Let $k: X \rightarrow A$ be a $\lambda_{(N, Y)}(\mathcal{F}, \mathcal{G})$-map and assume that $A$ is a subset of $W$. Since $f$ is $\mathcal{G}$-e-universal in $N$, there is a $V \in n(Y, U)$ such that $h \stackrel{\theta}{\approx} f$ for every $h \in \mathcal{G}_{X}^{V}$. Since $k$ is $(\mathcal{F}, \mathcal{G})$-e-liftable in $(N, P)$, there is an $R \in n(Y, U)$ such that for every $a \in \mathcal{F}_{A}^{R}$ there is a $b \in \mathcal{G}_{X}^{V}$ with $b \stackrel{\eta}{=} a \circ k$. Select an $s \in \mathcal{F}_{W}^{R}$ and a $t \in \mathcal{G}_{X}^{V}$ with $G \circ s \stackrel{\eta}{=} i_{W_{1} P}$ and $t \stackrel{\theta}{=}\left(\left.s\right|_{A}\right) \circ k$. Observe that $t \stackrel{\theta}{\approx} f$. Hence, $k=$ $i_{W, P} \circ k \stackrel{\eta}{=} G \circ\left(\left.s\right|_{A}\right) \circ k$ and $G \circ\left(\left.s\right|_{A}\right) \circ k \stackrel{\eta}{=} G \circ t \stackrel{\eta}{\approx} G \circ f \stackrel{\eta}{=} g \circ f$. It follows 
that $k \stackrel{\sigma}{\approx} g \circ f$.

A map $f$ is an $[\mathcal{F}, \mathcal{G}]$-e-progression in $Z$ provided for cvery $\sigma \in \bar{Z}$ and every $a \in \mathcal{F}_{X}^{Z}$ there is a $b \in \mathcal{G}_{Y}^{Z}$ with $a \stackrel{\sigma}{=} b \circ f$.

A map $f$ is an $(\mathcal{F}, \mathcal{G})$-e-progression in $(P, Z)$ provided for every $\sigma \in \tilde{P}_{Z}$ there is a $V \in n(Z, P)$ such that for every $a \in \mathcal{F}_{X}^{V}$ there is a $b \in \mathcal{G}_{Y}^{Z}$ with $a \stackrel{\sigma}{=} b \circ f$.

A map $f$ is an $(\mathcal{F}, \mathcal{G})$-e-progression in $(P, Z)$ provided for every $\sigma \in \tilde{P}_{Z}$ and every $U \in n(Z, P)$ there is a $V \in n(Z, P)$ such that for every $a \in \mathcal{F}_{X}^{V}$ there is a $b \in \mathcal{G}_{Y}^{U}$ with $a \stackrel{\sigma}{=} b \circ f$.

Observe that an ALI (approximately left invertible) map $f$ (i. e., a map such that for every $\sigma \in \tilde{X}$ there is a map $s: Y \rightarrow X$ with $\left.s \circ f \stackrel{o}{=} i d_{X}\right)$ is an $[\mathcal{A}, \mathcal{A}]$-e-progression in every space $Z$. Similarly, an ALI in $M$ map $f$ (i. e., a map such that for every $\sigma \in \tilde{M}_{X}$ and every $U \in n(X, M)$ there is a map $s: Y \rightarrow U$ with $s$ of $\left.\stackrel{o}{=} i_{X, U}\right)$ will be an $(\mathcal{A}, \mathcal{A})$-e-progression in every pair $(P, Z)$ where $P$ is an absolute neighborhhod retract. It will be an $(\mathcal{A}, \mathcal{A} \mid$-eprogression provided, in addition, $Z$ is an approximate polyhedron.

Theorem 7 .

(a) If $f$ is a surjective $[\mathcal{F}, \mathcal{G}]$-e-progression and $g$ is $\mathcal{G}$-universal, then $g \circ f$ is $\mathcal{F}$-universal.

(b) If $f$ is a surjective $(\mathcal{F}, \mathcal{G}$-e-progression in $(P, Z)$ and $g$ is $\mathcal{G}$-universal, then $g \circ f$ is $\mathcal{F}$-e-universal in $P$.

(c) If $f$ is a surjective $(\mathcal{F}, \mathcal{G})$-e-progression in $(P, Z)$ and $g$ is $\mathcal{G}$-e-universal in $P$, then $g \circ f$ is $\mathcal{F}$-e-universal in $P$.

Proof: (c). Let $\sigma \in \tilde{P}_{Z}$. Let $\eta \in \sigma^{*}$. Since $g$ is $\mathcal{G}$-e-universal in $P$, there is a $U \in n(Z, P)$ such that $g \stackrel{\eta}{\approx} h$ for every $h \in \mathcal{G}_{Y}^{U}$. Choose a $V \in n(Z, P)$ such that for every $a \in \mathcal{F}_{X}^{V}$ there is a $b \in \mathcal{G}_{Y}^{U}$ with $a \stackrel{\eta}{=} b \circ f$.

Let $k \in \mathcal{F}_{X}^{V}$. Select an $h \in \mathcal{G}_{Y}^{U}$ with $k \stackrel{n}{=} h \circ f$. By assumption, $g \stackrel{n}{\approx} h$. But, since $f$ is onto, the last relation implics $g \circ f \stackrel{\eta}{\approx} h \circ f$. Hence, $k \stackrel{\sigma}{\approx} g \circ f$.

The (b) part of the next theorem improves the (b) part of Theorem 8 in [10]. We replace Sanjurjo's assumption that a map $r$ is refinable with a weaker assumption (for example, that it is weakly refinable).

Theorem 8. Let $\mathcal{F}$ be a legal class of maps.

(a) If $f$ is $A R I[\mathcal{F}]$ and $g$ is $\mathcal{F}$-universal, then $g \circ f$ is $\mathcal{A}$-universal.

(b) If $f$ is $A R I[\mathcal{F})$ in $(M, N), g$ is $\mathcal{F}$-e-universal in $P$, and $P$ is an approximate polyhedron, then $g \circ f$ is $\mathcal{A}$-e-universal in $P$.

Proof: (b). Let $\sigma \in \tilde{P}_{Z}$. Let $\xi \in \sigma^{* *}$ and $\eta \in \xi^{*}$. Since $g$ is $\mathcal{F}$-e-universal in $P$, there is a $U \in n(Z, P)$ such that $g \stackrel{\eta}{\approx} h$ for every $h \in \mathcal{F}_{Y}^{U}$. We claim that $k \stackrel{\sigma}{\approx} g \circ f$ for every map $k: X \rightarrow U$. 
Indeed, let $k: X \rightarrow U$ be a map. Since $P$ is an approximate polyhedron, there is a $W \in n(X, M)$, a $V \in n(Y, N)$, and maps $K: W \rightarrow U$ and $G: V \rightarrow P$ such that $\left.k \stackrel{\underline{\xi}}{=} K\right|_{X}$ and $\left.g \stackrel{\underline{\xi}}{=} G\right|_{Y}$. Let $\theta=G^{-1}(\eta)$. Since $f$ is $A R I[\mathcal{F})$ in $(M, N)$, there is an $R \in n(X, W)$ and a map $F: R \rightarrow V$ such that $\left.f \stackrel{\theta}{=} F\right|_{X}$ and for every $T \in n(X, R)$ there is an $s \in \mathcal{F}_{Y}^{T}$ with $F \circ s \stackrel{\theta}{=} i_{Y, N}$.

Let $T \in n(X, R)$. Choose an $s \in \mathcal{F}_{Y}^{\gamma}$ with $F \circ s \stackrel{\theta}{=} i_{Y, N}$. Since the class $\mathcal{F}$ is legal, the composition $K \circ s: Y \rightarrow U$ is from $\mathcal{F}_{Y}^{U}$. By assumption, $g \stackrel{\eta}{\approx} K \circ s$. On the other hand, from $F \circ s \stackrel{\theta}{=} i_{Y, N}$ we get $g \stackrel{\underline{\eta}}{=} G \circ F \circ s$. Hence, we have $K \circ s \stackrel{\xi}{\approx} G \circ F \circ s$ and $\left.\left.K\right|_{T} \stackrel{\xi}{\approx} G \circ F\right|_{T}$. Since $T$ was arbitrary, $\left.\left.K\right|_{X} \stackrel{\xi}{\approx} G \circ F\right|_{X}$. But, $\left.G \circ F\right|_{X} \stackrel{\eta}{=} G \circ f \stackrel{\xi}{=} g \circ f$, and $\left.k \stackrel{\xi}{=} K\right|_{X}$ so that $k \stackrel{\sigma}{\approx} g \circ f$.

A map $f$ is $Z[\mathcal{F}, \mathcal{G}]$-e-movable provided for every $\sigma \in \bar{Y}$ and every $a \in \mathcal{F}_{Z}^{X}$ there is a $b \in \mathcal{G}_{Z}^{Y}$ with $b \stackrel{a}{=} f \circ a$.

A map $f$ is $Z\left(\mathcal{F}, \mathcal{G}\right.$ ]-e-movable in $(M, N)$ provided for every $\sigma \in \tilde{N}_{Y}$ there is a $U \in n(X, M)$ and a map $F: U \rightarrow N$ such that $\left.f \stackrel{\sigma}{=} F\right|_{X}$ and for every $a \in \mathcal{F}_{Z}^{U}$ there is a $b \in \mathcal{G}_{Z}^{Y}$ with $b \stackrel{\sigma}{=} F \circ a$.

A map $f$ is $Z(\mathcal{F}, \mathcal{G})$-e-movable in $(M, N)$ provided for every $\sigma \in \tilde{N}_{Y}$ there is a $U \in n(X, M)$ and a map $F: U \rightarrow N$ such that $\left.f \stackrel{\sigma}{=} F\right|_{X}$ and for every $a \in \mathcal{F}_{Z}^{U}$ and every $V \in n(Y, N)$ there is a $b \in \mathcal{G}_{Z}^{V}$ with $b \stackrel{o}{=} F \circ a$.

Observe that a map $f$ is $Z(\mathcal{A}, \mathcal{A})$-e-movable in $(Q, Q)$ iff $f$ is internally $Z$-e-movable [3]. Similarly, a map $f$ is $Z(\mathcal{A}, \mathcal{A})$-e-movable in $(Q, Q)$ iff $f$ is $Z$-e-movable [3].

Let $\mu Z[\mathcal{F}, \mathcal{G}]$ denote the class of all $Z[\mathcal{F}, \mathcal{G}]$-e-movable maps. The following notations $\mu_{(M, N)} Z(\mathcal{F}, \mathcal{G}]$ and $\mu_{(M, N)} Z(\mathcal{F}, \mathcal{G})$ have analogous meanings.

\section{Theorem 9.}

(a) If $f$ is $A R I[\mathcal{F}]$ and $g$ is $\mathcal{G}$-universal, then $g \circ f$ is $\mu Y[\mathcal{F}, \mathcal{G}]$-universal.

(b) If $f$ is $A R I[\mathcal{F})$ in $(M, N)$ and $g$ is both $\mathcal{G}$-universal and extendable in $(N, P)$, then $g$ of is $\mu_{(M, P)} Y(\mathcal{F}, \mathcal{G}]$-universal.

(c) If $f$ is $A R I(\mathcal{F})$ in $(M, N)$ and $g$ is both $\mathcal{G}$-e-universal and extendable in $(N, P)$, then $g \circ f$ is $\mu_{(M, P)} Y(\mathcal{F}, \mathcal{G})$-e-universal.

Proof: (b). Let a $\sigma \in \tilde{Z}$ and a $Y(\mathcal{F}, \mathcal{G})$-e-movable in $(M, P)$ map $k: X \rightarrow A$ with $A$ a subset of $Z$ be given. Select an $\eta \in \tilde{P}$ such that $\left.\eta\right|_{Z}$ refines $\sigma$. Let $\xi \in \eta^{*}$. Let $\nu \in \xi^{* *}$.

Since $k$ is $Y(\mathcal{F}, \mathcal{G}$-e-movable in $(M, P)$, there is a $U \in n(X, M)$ and a map $K: U \rightarrow P$ such that $\left.k \stackrel{\nu}{=} K\right|_{X}$ and for every $a \in \mathcal{F}_{Y}^{U}$ there a $b \in \mathcal{G}_{Y}^{A}$ with $b \stackrel{\nu}{=} K \circ a$.

Choose a $V \in n(Y, N)$ and a map $G: V \rightarrow P$ with $\left.g \stackrel{\xi}{=} G\right|_{Y}$. Let $\theta=$ $G^{-1}(\nu)$. Since $f$ is $A R I[\mathcal{F})$ in $(M, N)$, there is a $W \in n(X, U)$ and a map 
$F: W \rightarrow N$ such that $\left.f \stackrel{\theta}{=} F\right|_{X}$ and for every $L \in n(X, W)$ there is an $s \in \mathcal{F}_{Y}^{L}$ with $F \circ s \stackrel{\theta}{=} i_{Y, N}$.

Let $R \in n(X, W)$. By assumption, we know there is an $s \in \mathcal{F}_{Y}^{R}$ with $F \circ s \stackrel{\theta}{=} i_{Y, N}$. Choose a $t \in \mathcal{G}_{Y}^{A}$ with $t \stackrel{v}{=} K \circ s$. Since $g$ is $\mathcal{G}$-universal, $t \approx g$. Now, $K \circ s \stackrel{\nu}{=} t \stackrel{\nu}{\approx} g$ and $\left.G\right|_{Y} \stackrel{\nu}{=} G \circ F \circ s$. It follows that $\left.\left.K\right|_{R} \stackrel{\xi}{\approx} G \circ F\right|_{R}$. But, since $R$ is arbitrary, we get $\left.\left.K\right|_{X} \stackrel{\xi}{\approx} G \circ F\right|_{X}$. Hence, $k \stackrel{\sigma}{\approx} g \circ f$.

(c). Let $\sigma \in \tilde{P}_{Z}$. Let $\eta \in \sigma^{*}$ and $\xi \in \eta^{* *}$. By asumptions on $g$, there is a $J \in n(Z, P)$, a $V \in n(Y, N)$, and a map $G: V \rightarrow P$ such that $g \stackrel{\xi}{\approx} h$ for evcry $h \in \mathcal{G}_{Y}^{J}$ and $\left.g \stackrel{\xi}{=} G\right|_{Y}$. Let $\theta=G^{-1}(\xi)$.

Consider a $Y(\mathcal{F}, \mathcal{G})$-e-movable in $(M, P)$ map $k: X \rightarrow A$ and assume that $A$ is a subset of $J$. Select a $U \in n(X, M)$ and a map $K: U \rightarrow P$ such that $\left.k \stackrel{\underline{\xi}}{=} K\right|_{X}$ and for every $a \in \mathcal{F}_{Y}^{U}$ and every $L \in n(Z, P)$ there is a $b \in \mathcal{G}_{Y}^{L}$ with $\left.b \stackrel{\xi}{=} K\right|_{A}$. Since $f$ is $A R I[\mathcal{F})$ in $(M, N)$, there is a $W \in n(X, U)$ and a map $F: W \rightarrow P$ such that $\left.f \stackrel{\theta}{=} F\right|_{X}$ and for every $R \in n(X, W)$ there is an $s \in \mathcal{F}_{Y}^{R}$ and a $t \in \mathcal{G}_{Y}^{J}$ with $t \stackrel{\xi}{=} K \circ s$ and $F \circ s \stackrel{\theta}{=} i_{Y, N}$. The way in which $J$ was selected implies $t \stackrel{\xi}{\approx} g$. Now, we have the following chain of relations $\left.K \circ s \stackrel{\underline{\xi}}{=} t \stackrel{\xi}{\approx} g \stackrel{\xi}{=} G\right|_{Y} \stackrel{\xi}{=} G \circ F \circ s$. It follows that $\left.\left.K\right|_{R} \stackrel{\eta}{\approx} G \circ F\right|_{R}$. Since $R$ was arbitrary, we get $\left.\left.K\right|_{X} \stackrel{\eta}{\approx} G \circ F\right|_{X}$. Hence, $k \stackrel{\sigma}{\approx} g \circ f$.

We shall now establish partial converses of the above theorems. This tirne we assume that the composition $g \circ f$ is universal and try to get that either $g$ or $f$ is universal.

\section{Theorem 10.}

(a) If $g \circ f$ is $\mathcal{F}$-universal and $g$ is both an embedding and $X[\mathcal{F}, \mathcal{G}]-\varepsilon$ movable, then $f$ is $\mathcal{G}$-universal.

(b) If $g \circ f$ is $\mathcal{F}$-universal and $g$ is both an embedding and $X(\mathcal{F}, \mathcal{G}]$-e-movable in $(N, P)$, then $f$ is $\mathcal{G}$-e-universal in $N$.

(c) If $g \circ f$ is $\mathcal{F}$-e-universal in $P$ and $g$ is both an embedding and $X(\mathcal{F}, \mathcal{G})$ $e$-movable in $(N, P)$, then $f$ is $\mathcal{G}$-e-universal in $N$.

Proof: (c). Let $\sigma \in \bar{N}_{Y}$. Since $g$ is an embedding, there is a $\xi \in \tilde{P}_{Z}$ such that for every $U \in n(Y, N)$ and every map $G: U \rightarrow P$ with $\left.g \stackrel{\xi}{=} G\right|_{Y}$ there is a $V \in n(Y, U)$ such that $G(x) \stackrel{\xi}{=} G(y)$ for $x, y \in V$ implies $x \stackrel{g}{=} y$. Let $\eta \in \xi^{*}$. We use now the sccond assumption on $g$ to choose a $U \in n(Y, N)$ and a map $G: U \rightarrow P$ such that $\left.g \stackrel{\eta}{=} G\right|_{Y}$ and for every $h \in \mathcal{G}_{X}^{U}$ and every $W \in n\{Z, P)$ there is a $k \in \mathcal{F}_{X}^{W}$ with $k \stackrel{\eta}{=} G \circ h$. Since $g \circ f$ is $\mathcal{F}$-e-universal in $P$, there is a $W \in n(Z, P)$ such that $k \stackrel{\eta}{\approx} g \circ f$ for every $k \in \mathcal{F}_{X}^{W}$. Finally, pick a $V \in n(Y, U)$ using the way in which $\xi$ was selected. 
Let $h \in \mathcal{G}_{X}^{V}$. Choose a $k \in \mathcal{F}_{X}^{W}$ with $k \stackrel{\eta}{=} G \circ h$. By assumption, $k \stackrel{\eta}{\approx} g \circ f$ and $g \circ f \stackrel{\eta}{=} G \circ f$. Hence, $G \circ f \stackrel{\xi}{\approx} G \circ h$ and $f \stackrel{\sigma}{\approx} h$.

Theorem 11. Let $\mathcal{F}$ be a solid class of maps.

(a) If $g \circ f$ is $\mathcal{F}$-universal and $g$ is both an embedding and an $[\mathcal{F}]$ map, then $f$ is $\mathcal{A}$-universal.

(b) If $g \circ f$ is $\mathcal{F}$-e-universal in $P$ and $g$ is both an embedding and an $[\mathcal{F}, P)$-map, then $f$ is $\mathcal{A}$-universal.

(c) If $g \circ f$ is $\mathcal{F}$-e-universal in $P$ and $g$ is both an embedding and an $(\mathcal{F}, N, P)-$ map, then $f$ is $\mathcal{A}$-e-universal in $N$.

Proof: (c). Let $\sigma \in \tilde{N}_{Y}$. Select $\xi$ and $\eta$ as in the proof of the previous theorem. Since $g \circ f$ is $\mathcal{F}$-e-universal in $P$, there is a $V \in n(Z, P)$ such that $k \stackrel{\eta}{\approx} g \circ f$ for every $k \in \mathcal{F}_{X}^{V}$. By the second assumption on $g$, there is a $U \in$ $k n(Y, N)$ and a $G \in \mathcal{F}_{U}^{V}$ with $\left.g \stackrel{\eta}{=} G\right|_{Y}$. Let a $W \in n(Y, U)$ be selected using the way in which $\xi$ was chosen.

Let $h: X \rightarrow W$ be a map. Since $\mathcal{F}$ is a solid class of maps, $G \circ h \in \mathcal{F}_{X}^{V}$. It follows that $G \circ h \stackrel{\eta}{\approx} g \circ f \stackrel{\eta}{=} G \circ f$. Hence, $G \circ h \stackrel{\xi}{\approx} G \circ f$ and $h \stackrel{\sigma}{\approx} f$.

Theorem 12. Let $\mathcal{F}$ be a solid class of maps.

(a) If $g \circ f$ is $\mathcal{G}$-universal and $g$ is both an embedding and on $[\mathcal{F}]$-map, then $f$ is $\lambda_{Z}[\mathcal{F}, \mathcal{G}]$-universal.

(b) If $g \circ f$ is $\mathcal{G}$-universal and $g$ is both an embedding and on $[\mathcal{F}, P)-$ map, then $f$ is $\lambda_{(P, Z)}(\mathcal{F}, \mathcal{G}]$-universal.

(c) If $g \circ f$ is $\mathcal{G}$-e-universal in $P$ and $g$ is both an embedding and an $[\mathcal{F}, P)$-map, then $f$ is $\lambda_{(P, Z)}(\mathcal{F}, \mathcal{G})$-universal.

Proof: $(c)$. Let $h: X \rightarrow A$ be an $(\mathcal{F}, \mathcal{G})$-e-liftable in $(P, Z)$ map and assume that $A$ is a subset of $Y$. In order to show that $f \approx h$ it suffices to see that $f \stackrel{\sigma}{\approx} h$ for every $\sigma \in \check{Y}$.

Let $\sigma \in \dot{Y}$. Since $g$ is an embedding, there is a $\xi \in \tilde{Z}$ such that $g(x) \stackrel{\xi}{=} g(y)$ for $x, y \in Y$ implies $x \stackrel{o}{=} y$. Let $\eta \in \tilde{P}$ has the property that $\left.\eta\right|_{Z}$ refincs $\xi$. Let $\mu \in \eta^{*}$. By assumption on $g \circ f$, there is a $U \in n(Z, P)$ with $k \stackrel{\mu}{\approx} g \circ f$ for every $k \in G_{X}^{U}$. Since $h$ is $(\mathcal{F}, \mathcal{G})$-e-liftable in $(P, Z)$, there is a $V \in n(Z, U)$ such that for every $a \in \mathcal{F}_{A}^{V}$ there is a $b \in \mathcal{G}_{X}^{U}$ with $b \stackrel{\mu}{=} a \circ h$. By the second assumption on $g$, there is an $m \in \mathcal{F}_{Y}^{V}$ such that $\left.g \stackrel{\mu}{=} m\right|_{Y}$.

The restriction $\left.m\right|_{A}$ is in $\mathcal{F}_{A}^{V}$ because the class $\mathcal{F}$ is solid. It follows that there is a $k \in \mathcal{G}_{X}^{U}$ with $k \stackrel{\mu}{=} m \circ h$. But, $k \stackrel{\mu}{\approx} g \circ f$. Hence, $g \circ h \cong g \circ f$ and $h \stackrel{\sigma}{\approx}$. 
Theorem 13.

(a) If $g \circ f$ is $\mathcal{F}$-universal and $f$ is $[\mathcal{F}, \mathcal{G}]$-e-liftable in $Z$, then $g$ is $\mathcal{G}$ universal.

(b) If $g \circ f$ is $\mathcal{F}$-e-universal in $P$ and $f$ is $[\mathcal{F}, \mathcal{G})$-e-liftable in $(P, Z)$, then $g$ is $\mathcal{G}$-universal.

(c) If $g \circ f$ is $\mathcal{F}$-universal in $P$ and $f$ is $(\mathcal{F}, \mathcal{G}]$-e-liftable in $(P, Z)$, then $g$ is $\mathcal{G}$-e-universal in $P$.

(d) If $g \circ f$ is $\mathcal{F}$-e-universal in $P$ and $f$ is $(\mathcal{F}, \mathcal{G})$-e-liftable in $(P, Z)$, then $g$ is $\mathcal{G}$-e-universal in $P$.

Proof: $(d)$. Let $\sigma \in \tilde{P}_{Z}$. Let $\xi \in \sigma^{*}$. Since $g \circ f$ is $\mathcal{F}$-e-universal in $P$, there is a $U \in n(Z, P)$ such that $k \stackrel{\xi}{\approx} g \circ f$ for every $k \in \mathcal{F}_{X}^{U}$. By assumption on $f$, there is a $V \in n(Z, U)$ such that for every $a \in \mathcal{G}_{Y}^{V}$ there is a $b \in \mathcal{F}_{X}^{U}$ with $b \stackrel{\xi}{=} a \circ f$.

Let $h \in \mathcal{G}_{Y}^{V}$. Choose a $k \in \mathcal{F}_{X}^{U}$ with $k \stackrel{\xi}{=} h \circ f$. Observe that $k \stackrel{\xi}{\approx} g \circ f$. Hence, $h \circ f \stackrel{\xi}{=} k \stackrel{\xi}{\approx} g \circ f$ and $h \stackrel{\sigma}{\approx} g$.

Theorem 14. Let $\mathcal{F}$ be a legal class of maps.

(a) If $g \circ f$ is $\mathcal{F}$-universal and $f$ is an $[\mathcal{F}]$-map, then the mapping $g$ is A-universal.

(b) If $g \circ f$ is $\mathcal{F}$-e-universal in $P, f$ is an $[\mathcal{F}, N)$-map, and $P$ is an approximate polyhedron, then $g$ is $\mathcal{A}$-e-universal in $P$.

Proof: (b). Let $\sigma \in \tilde{P}_{Z}$. Let $\xi \in \sigma^{*}$. Since $g \circ f$ is $\mathcal{F}$-e-universal in $P$, there is a $U \in n(Z, P)$ such that $k \stackrel{\xi}{\approx} g \circ f$ for every $k \in \mathcal{F}_{X}^{U}$.

Let $h \in \mathcal{A}_{Y}^{U}$. Since $P$ is an approximate polyhedron, there is a $V \in n(Y, N)$ and a continuous function $H: V \rightarrow U$ with $\left.h \stackrel{\underline{\xi}}{=} H\right|_{Y}$. Let $\theta=H^{-1}(\xi)$. Choose an $a \in \mathcal{F}_{X}^{V}$ with $a \stackrel{\theta}{=} f$. The assumptions about $\mathcal{F}$ and $U$ imply $H \circ a \in \mathcal{F}_{X}^{U}$ and $g \circ f \stackrel{\xi}{\approx} H \circ a$. But, $H \circ a \stackrel{\xi}{=} H \circ f \stackrel{\underline{\xi}}{=} h \circ f$. Hence, $g \stackrel{\sigma}{\approx} h$.

\section{Theorem 15.}

(a) If $f$ is an $[\mathcal{F}]$-map and $g \circ f$ is $\mathcal{G}$-universal, then $g$ is $\mu X[\mathcal{F}, \mathcal{G}]$-universal.

(b) If $f$ is an $[\mathcal{F}, N)$-map and $g \circ f$ is $\mathcal{G}$-universal, then the mapping $g$ is $\mu_{(N, P)} X(\mathcal{F}, \mathcal{G}]$-universal.

(c) If $f$ is an $[\mathcal{F}, N)$-map and $g \circ f$ is $\mathcal{G}$-e-universal in $P$, then $g$ is $\mu_{(N, P)} X(\mathcal{F}, \mathcal{G})$-e-universal.

Proof: (c). Let $\sigma \in \tilde{P}_{Z}$. Let $\xi \in \sigma^{* *}$. Since $g \circ f$ is $\mathcal{G}$-e-universal in $P$, there is a $U \in n(Z, P)$ such that $k \stackrel{\xi}{\approx} g \circ f$ for every $k \in \mathcal{G}_{X}^{J}$. 
Consider an $X(\mathcal{F}, \mathcal{G})$-e-movable in $(N, P)$ map $h: Y \rightarrow A$ and assume that $A$ is contained in $U$. Choose a $V \in n(Y, N)$ and a map $H: V \rightarrow P$ such that $\left.h \stackrel{\xi}{=} H\right|_{Y}$ and for every $a \in \mathcal{F}_{X}^{V}$ and every $W \in n(Z, P)$ there is a $b \in \mathcal{G}_{X}^{W}$ with $b \stackrel{\xi}{=} H \circ a$. Let $\theta=H^{-1}(\xi)$. Since $f$ is an $[\mathcal{F}, N)$-map, there is an $a \in \mathcal{F}_{X}^{V}$ with $a \stackrel{\theta}{=} f$. By assumption, there is a $b \in \mathcal{G}_{X}^{W}$ with $b \stackrel{\underline{\xi}}{H} H \circ a$ and $b \stackrel{\xi}{\approx} g \circ f$. Hence, $g \circ f \stackrel{\xi}{\approx} b$ and $b \stackrel{\underline{\xi}}{=} H \circ a \stackrel{\xi}{=} H \circ f \stackrel{\xi}{=} h \circ f$ and $g \stackrel{\sigma}{\approx} h$.

\section{References}

1. F. Al-MUSAllam and S.B. NADLER, IR., Almost universal maps and the almost fixed point property, in "Differential geometry, calculus of variations, and their applications," Lecture Notes in Pure and Appl. Math. 100, Dekker, New York, 1985, pp. 19-35.

2. K. Borsuk, On nearly extendable maps, Bull. Acad. Polon. Sci., Sér. sci. math., astronom. et phys. 23 (1975), 753-760.

3. Z. C̈ERIN, On global properties of maps I, Suppl. Rendiconti Circ. Mat. Palermo 12 (1986), 19-45.

4. G. Gauthier, La théorie des retracts approximatifs et le théorème des points fixes de Lefschetz, Dissertationes Math. 217 (1983), 1-78.

5. W. HOLSZTYŃSKI, Une généralisation du théorème de Brouwer sur les points invariants, Bull. Acad. Polon. Sci., Sér. sci. math., astronom. et phys. 12 (1964), 603-606.

6. W. HoLSZTYŃSKI, Universal mappings and fixed point theorems, Bull. Acad. Pol. Sci., Sér. sci. math., astronom. et phys. 15 (1967), 433-438.

7. W. HOLSZTYNSKI, On the composition and products of universal mappings, Fund. Math. 64 (1969), 181-188.

8. G. KOZLOWSKI, Images of ANR's, Trans. Amer. Math. Soc. (to appear).

9. S. MARDEŠIĆ, Approximate polyhedra, resolutions of maps, and shape fibrations, Fund. Math. 114 (1981), 53-78.

10. J. M. R. SANJuRJo, Stability of the fixed point property and universal maps, Proc. Amer. Math. Soc. 105 (1989), 221-230.

Department of Mathemalics

University of Zagreb

41001 Zagreb, p.p. 187

CROATIA

Rebut el 24 d'Abril de 1990 